\title{
Construção de cartas centílicas da coordenação motora de crian- ças dos 6 aos 11 anos da Região Autónoma dos Açores. Portugal
}

\author{
Sónia Moreira Vidal ${ }^{1}$ \\ Alcibíades Bustamante ${ }^{1,2}$ \\ Vítor Pires Lopes ${ }^{3}$ \\ André Seabra ${ }^{1,4}$ \\ Rui Garganta da Silva1,4 \\ José António Maia ${ }^{1,4}$
}

https://doi.org/10.5628/rpcd.09.01.24

\author{
${ }^{1}$ Laboratório de Cineantropometria e de Estatística Aplicada \\ Faculdade de Desporto, Universidade do Porto, Portugal \\ 2 Universidade Nacional de Educación "Enrique Guzmán y Valle" \\ La Cantuta, Lima, Perú \\ ${ }^{3}$ Departamento de Ciências do Desporto, Instituto \\ Politécnico de Bragança, Portugal \\ ${ }^{4}$ Centro de Investigação, Formação, Inovação e Intervenção \\ em Desporto (CIFI $\left.{ }^{2} \mathrm{D}\right)$, Faculdade de Desporto, \\ Universidade do Porto, Portugal
}

\begin{abstract}
RESUMO
Objectivo: Construir cartas centílicas e respectiva distribuição de valores da Coordenação Motora em crianças açorianas dos 6 aos 11 anos segundo o género e idade.

Metodologia: A amostra é constituída por 2359 meninas e 2365 meninos da Região Autónoma dos Açores. A Coordenação Motora foi avaliada através da bateria KTK, que compreende quatro provas: equilíbrio à retaguarda, saltos laterais, saltos monopedais e transposição lateral. As estatísticas descritivas básicas foram calculadas no SPSS 15. os centis foram estimados pelo método da máxima verosimilhança no software LMS versão 1.32 e as cartas centílicas construídas no Excell.

Resultados: Em todas as provas da bateria de testes KTK, para ambos os sexos, é visível um incremento do desempenho quer dos valores médios quer para categorias extremas de performance, seja o P3 ou P10, ou ainda os P90 e P97, não obstante uma forte variação em cada valor discreto de idade e sexo. Conclusões: Com base nos valores centílicos do desempenho da Coordenação Motora pode traçar-se perfis configuracionais e interpretar-se o seu significado relativamente ao que é esperado para uma dada idade e ano de escolaridade. Discorre daqui o contributo deste estudo em termos pedagógicos para a disciplina de Actividade Física e Desportiva no $1^{\circ}$ ciclo do Ensino Básico.
\end{abstract}

Palavras-chave: coordenação motora, cartas centílicas, educação física

\section{ABSTRACT \\ Motor coordination centile charts of children aged 6 to 11 years in the Azores Autonomous Region, Portugal}

Purpose: The aim of this study was to produce centile curves of Motor Coordination and the distribution of their values in Azorean children aged 6 to 11 years according to age and sex.

Methodology: Sample size comprises 2359 girls and 2365 boys of the Autonomous Region of the Azores; they were evaluated with the KTK battery, which includes four tests: backward balance, jumping sideways, hopping on one leg and shifting platforms. Basic descriptive statistics were calculated on SPSS 15. We used LMS software version 1.32 compute centile using maximum likelihood, and Excell to construct the centile curves.

Results: Both sexes showed visible increase in performance not only in mean values but also in extreme performance, described by such percentiles as P3, P10, P90 or P97.

Conclusions: Motor Coordination performance percentile values may be used to portray individual profiles and to interpret their meaning according to what is expected for a given age and scholar level. Hence the contribution of this study in terms of teaching purposes for Physical Education in primary schools.

Key-words: motor coordination, centiles curves, physical education 


\section{INTRODUÇÃO}

$\mathrm{O}$ interesse pelo estudo de diferentes facetas do desenvolvimento motor de crianças, sobretudo no que diz respeito à sua coordenação motora (CoM), tem ganho algum destaque sobretudo em termos pedagógicos e clínicos $(9,22,21,19,20,25,31,12,3)$.

A pertinência do estudo da CoM está directamente relacionada com as mudanças verificadas ao longo do tempo nas mais variadas facetas e expressões de comportamento motor das crianças e da sua interacção contínua em factores mutáveis, ou não, do seu ambiente. As crianças e jovens são cada vez mais sedentárias( ${ }^{(2)}$, assumindo estilos de vida dependentes das novas tecnologias, robotização e meios de transporte motorizados. A insuficiência de Actividade Física e Desportiva (AFD) em quantidade e qualidade (nas idades de escolaridade básica) origina problemas de coordenação que irão reflectir-se negativamente no processo de aprendizagem motora, assim como no desenvolvimento dos mais variados padrões motores e capacidades cognitivas $(34,30)$. Daqui que não sejam de estranhar as posições oficiais de educadores nos grandes objectivos dos programas de Educação Física neste nível de ensino. Em Portugal, por exemplo, o Programa Oficial de AFD para o $1^{\circ}$ Ciclo do Ensino Básico (CEB), aprovado pelo Ministério de Educação, expõe entre outras as seguintes finalidades: "Na perspectiva da qualidade de vida, da saúde e do bem-estar: (1) melhorar a aptidão física, elevando as capacidades físicas de modo harmonioso e adequado às necessidades de desenvolvimento do aluno; (2) promover a aprendizagem de conhecimentos relativos aos processos de elevação e manutenção das capacidades físicas; (3) promover o gosto pela prática regular das actividades físicas e aprofundar a compreensão da sua importância como factor de saúde ao longo da vida e componente da cultura, quer na dimensão individual, quer social..."(17). A Escola é a instituição com maior encargo na implementação destas directivas, que na tentativa de desenvolver hábitos de vida activa e desportiva nas crianças e jovens depara-se muitas vezes com diversas dificuldades, como é o caso dos espaços e materiais inexistentes ou inadequados, o tempo útil de prática desportiva que é insuficiente, turmas com elevado número de alunos, entre outros, dificultando o cumprimento dos programas e o alcance das finalidades mencionadas.
Estranhamente, no mesmo programa, nada é referido de forma explícita o desenvolvimento das capacidades coordenativas que tão importantes são nas idades dos alunos do $1^{\circ} \mathrm{CEB}$, pois estas têm interferência no ritmo, assim como no modo de aprendizagem das técnicas desportivas, sua posterior estabilização e utilização em diversas situações ${ }^{(14)}$.

Preocupações de carácter pedagógico e clínico têm condicionado a investigação no âmbito da CoM com o intuito de obter informações mais detalhadas acerca das mudanças que ocorrem no tempo, dos predictores responsáveis pela alteração nas médias e variabilidade interindividual, sua relação com a formação integral dos alunos, bem como com a associação da qualidade do movimento à presença ou ausência de insuficiências coordenativas. Praticamente toda a pesquisa realizada no domínio da CoM a partir do uso da bateria de testes KTK (Körperkoordination Test für Kinder) tem sido de natureza experimental ou quase experimental(22, 29,35), descritiva e inferen$\operatorname{cial}^{(9,8,1,3)}$, nalguns casos predictiva ${ }^{(26)}$ e raras vezes longitudinal $(18,33)$. Estes estudos oriundos dos Estados Unidos da América, Alemanha, Brasil, Portugal ou Perú têm centrado a sua atenção em amostras de dimensão muito díspar cuja aplicabilidade local é da maior relevância em termos educativos. Contudo, nunca abordaram o problema da validade transcultural dos valores normativos dos autores alemães ${ }^{(18)}$ tão pouco construíram cartas centílicas do desenvolvimento da CoM. Uma das formas mais interessantes de apresentar o comportamento dos valores da CoM das crianças é a partir de cartas centílicas, que permitem especificar valores de referência, traçar perfis multidimensionais das crianças, e posicioná-las em termos centílicos relativamente a uma população estudada, de acordo com a idade e sexo. A relevância clínica e epidemiológica de cartas centílicas do crescimento estatuto-ponderal é por demais evidente, e nada obsta, bem pelo contrário, que o mesmo pensamento e abordagem quantitativa sejam aplicados à CoM.

A inexistência de valores de referência portugueses relativos à CoM conduziu à presente investigação, cujo propósito foi o de construir cartas centílicas e respectiva distribuição de valores da CoM segundo o sexo e idade a partir da bateria de testes KTK numa amostra representativa de crianças com idades compreendidas entre os 6 e os 11 anos. 


\section{MATERIAL E MÉTODOS \\ Amostra}

A amostra global é constituída por dois sub-conjuntos de dados. Um primeiro proveniente de um estudo transversal realizado na Região Autónoma dos Açores (RAA) (23). O segundo, realizado também na mesma região, contendo informação longitudinal de crianças que foram seguidas consecutivamente durante quatro anos(26). Enquanto que o primeiro sub-conjunto amostrou crianças de 8 de 9 ilhas, no segundo foram seleccionadas crianças de 4 ilhas. Os resultados da avaliação coordenativa referem-se a crianças dos 6 aos 11 anos de idade, sendo que os 6 anos correspondem a valores de idade decimal compreendidos entre os 6.00 e os 6.99; nas restantes idades, o intervalo considerado é o mesmo. O total de observações em cada uma das provas da bateria KTK e em cada sexo está mencionado no Quadro 1.

Quadro 1. Efectivo total de observações por prova e sexo.

\begin{tabular}{lcc}
\hline Prova & Feminino $(\mathrm{n})$ & Masculino $(\mathrm{n})$ \\
\hline Equilíbrio à retaguarda (ER) & 2356 & 2365 \\
\hline Salto Monopedal (SM) & 2321 & 2324 \\
\hline Salto Lateral (SL) & 2359 & 2360 \\
\hline Transposição Lateral (TL) & 2355 & 2358 \\
\hline
\end{tabular}

\section{Avaliação da coordenação motora}

A CoM é uma estrutura multidimensional que não pode ser medida directamente, daí que seja de elevada importância utilizar na sua avaliação uma bateria de provas que manifeste uma adequada operacionalização( ${ }^{(9)}$. Os autores alemães ${ }^{(18)}$ desenvolveram a bateria de testes KTK para identificar crianças com dificuldades de coordenação entre os cinco e os catorze anos de idade. Após vários estudos de análise factorial exploratória, identificaram um factor denominado como coordenação corporal, que continha os quatro testes actuais da bateria KTK:

1. Equilíbrio em marcha à retaguarda (ER), cujo objectivo é caminhar à retaguarda sobre três tábuas de diferentes larguras. É contado o número de passos que o aluno dá em cada uma delas sem apoiar o pé no chão. O resultado é igual ao somatório dos apoios realizados; 2 . Salto monopedais (SM), que consiste saltar a um pé (inicia com o pé preferido) por cima de uma ou mais placas de espuma sobrepostas, colocadas transversalmente na direcção do salto. A recepção é realizada com o pé com que iniciou o salto, não podendo tocar com o outro no chão; 3. Saltos Laterais (SL), que corresponde a saltos efectuados lateralmente, com ambos os pés, devendo manter-se juntos, durante 15 segundos. Conta-se o número de saltos; 4. Transposição Lateral (TL), que consiste na transposição lateral das plataformas durante 20 segundos, tantas vezes quanto possível. Conta-se o número de transposições durante o tempo limite.

No presente estudo foi considerado o desempenho em cada prova. Em momento algum se recorreu ao seu valor estandardizado pelos autores alemães, tão pouco se calculou o quociente motor. Esta decisão radicou no facto de não existir qualquer estudo que tenha mostrado a validade transcultural da pontuação sugerida pelos autores alemães dos resultados de cada prova.

\section{Procedimentos estatísticos}

Utilizaram-se os programas FileMaker Pro e Microsoft EXCEL para introdução e controlo de dados. Realizou-se a análise exploratória dos resultados com o propósito de avaliar a normalidade das distribuições e identificar a presença de "outliers" no programa estatístico SPSS 15.0. Diferentes aspectos de controlo da qualidade dos dados são referidos no primeiro trabalho realizado na RAA(23).

As cartas centílicas foram construídas com a ajuda do modelo matemático-estatístico LMS $(4,5)$, implementado no software LMS versão 1.32 (A program for calculating age-related referente centiles). Muito genericamente, o método de LMS sumaria três curvas ( $L=$ transformações de Box-Cox; $M=$ mediana e $S=$ coeficiente de variação), que são suavizadas pelo uso de cubic splines estimando parâmetros pelo método de máxima verosimilhança.

\section{RESULTADOS}

Nos Quadros 2, 3, 4 e 5 apresentam-se os valores de LMS e respectivos centis (P3, P10, P25, P50, P75, P90, P97) de cada uma das quatro provas do KTK para ambos os sexos e ao longo da idade (dos 6 aos 11 anos de idade de crianças açorianas). As cartas 
Quadro 2. Valores de LMS da prova ER de crianças açorianas de ambos os sexos.

\begin{tabular}{|c|c|c|c|c|c|c|c|c|c|c|c|}
\hline \multirow[t]{2}{*}{ Sexo } & \multirow[t]{2}{*}{ Idade } & \multirow[t]{2}{*}{$L$} & \multirow[t]{2}{*}{$M$} & \multirow[t]{2}{*}{$S$} & \multicolumn{7}{|c|}{ Percentis } \\
\hline & & & & & 3 & 10 & 25 & 50 & 75 & 90 & 97 \\
\hline \multirow[t]{6}{*}{ Meninos } & $6+$ & 0,53 & 22,52 & 0,49 & 5,49 & 9,95 & 15,64 & 22,52 & 30,57 & 39,78 & 50,12 \\
\hline & $7+$ & 0,76 & 30,77 & 0,43 & 7,70 & 14,54 & 22,29 & 30,77 & 39,87 & 49,49 & 59,60 \\
\hline & $8+$ & 0,92 & 37,39 & 0,36 & 11,41 & 19,81 & 28,49 & 37,39 & 46,47 & 55,68 & 65,02 \\
\hline & $9+$ & 1,09 & 42,32 & 0,31 & 14,85 & 24,31 & 33,43 & 42,32 & 51,04 & 59,62 & 68,09 \\
\hline & $10+$ & 1,21 & 45,22 & 0,29 & 16,89 & 27,00 & 36,35 & 45,22 & 53,73 & 61,97 & 69,99 \\
\hline & $11+$ & 1,23 & 45,66 & 0,28 & 17,22 & 27,43 & 36,81 & 45,66 & 54,13 & 62,31 & 70,25 \\
\hline \multirow[t]{6}{*}{ Meninas } & $6+$ & 0,50 & 25,25 & 0,49 & 6,28 & 11,20 & 17,53 & 25,25 & 34,35 & 44,85 & 56,73 \\
\hline & $7+$ & 0,66 & 29,93 & 0,43 & 8,29 & 14,47 & 21,73 & 29,93 & 38,99 & 48,85 & 59,43 \\
\hline & $8+$ & 0,86 & 36,97 & 0,36 & 12,15 & 19,99 & 28,29 & 36,97 & 45,93 & 55,15 & 64,59 \\
\hline & $9+$ & 1,05 & 41,54 & 0,31 & 14,99 & 24,01 & 32,84 & 41,54 & 50,15 & 58,67 & 67,13 \\
\hline & $10+$ & 1,19 & 44,93 & 0,29 & 16,46 & 26,59 & 35,99 & 44,93 & 53,53 & 61,86 & 69,98 \\
\hline & $11+$ & 1,23 & 45,64 & 0,29 & 16,72 & 27,12 & 36,66 & 45,64 & 54,23 & 62,52 & 70,56 \\
\hline
\end{tabular}

Figura 1. Curvas centílicas relativas à prova $E R$ de crianças açorianas dos 6 aos 11 anos de idade.
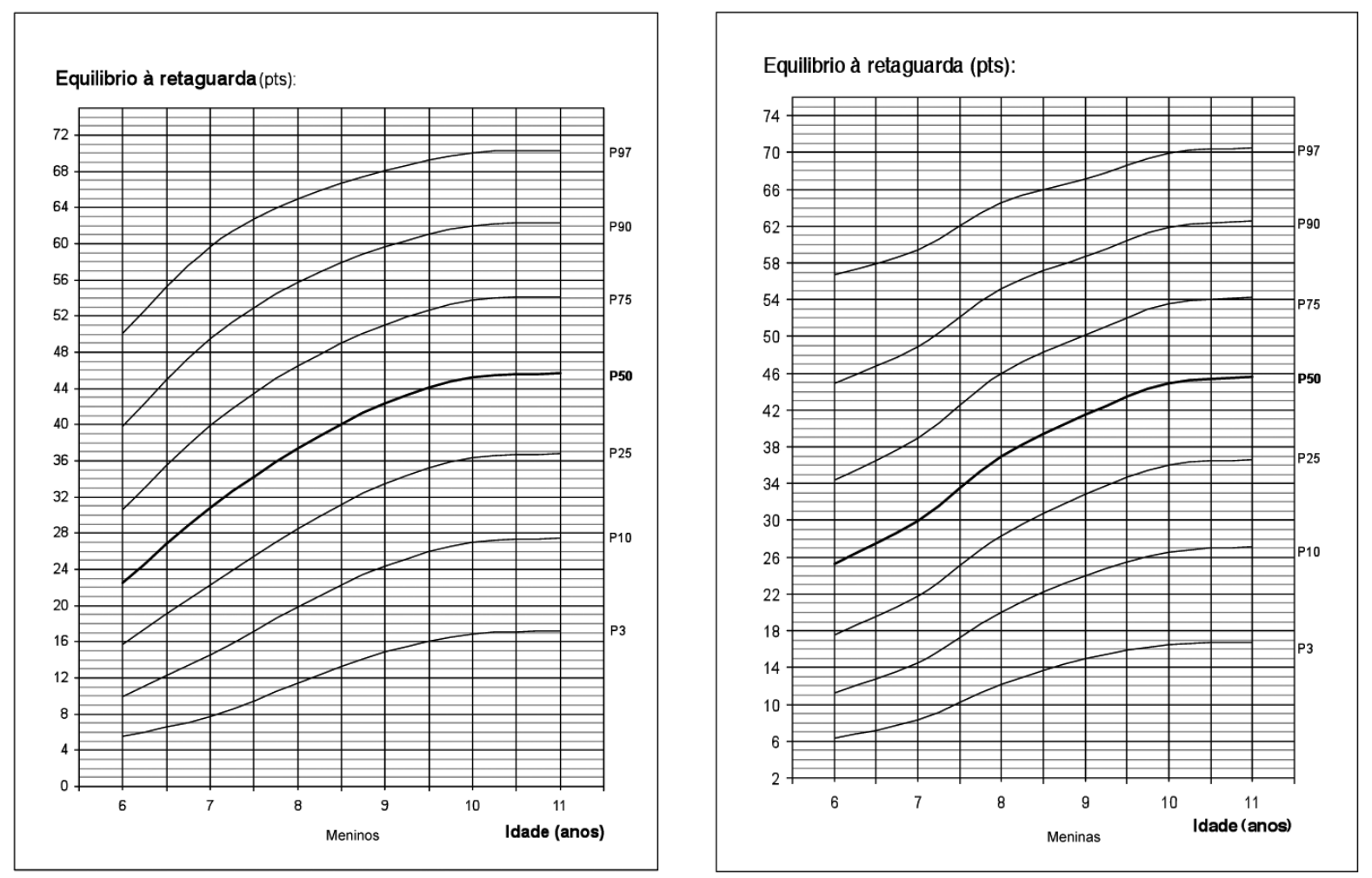
Quadro 3. Valores de LMS da prova SL de crianças açorianas de ambos os sexos.

\begin{tabular}{|c|c|c|c|c|c|c|c|c|c|c|c|}
\hline \multirow[t]{2}{*}{ Sexo } & \multirow[t]{2}{*}{ Idade } & \multirow[t]{2}{*}{$\mathrm{L}$} & \multirow[t]{2}{*}{$M$} & \multirow[t]{2}{*}{$S$} & \multicolumn{7}{|c|}{ Percentis } \\
\hline & & & & & 3 & 10 & 25 & 50 & 75 & 90 & 97 \\
\hline \multirow[t]{6}{*}{ Meninos } & $6+$ & 0,38 & 29,29 & 0,29 & 14,94 & 19,07 & 23,85 & 29,29 & 35,44 & 42,33 & 49,98 \\
\hline & $7+$ & 0,43 & 32,29 & 0,29 & 16,31 & 20,95 & 26,27 & 32,29 & 39,02 & 46,51 & 54,74 \\
\hline & $8+$ & 0,56 & 38,72 & 0,29 & 19,24 & 25,07 & 31,56 & 38,72 & 46,51 & 54,93 & 63,97 \\
\hline & $9+$ & 0,87 & 46,70 & 0,26 & 23,51 & 31,00 & 38,75 & 46,70 & 54,83 & 63,13 & 71,56 \\
\hline & $10+$ & 1,17 & 51,91 & 0,23 & 27,24 & 35,78 & 43,97 & 51,91 & 59,63 & 67,19 & 74,60 \\
\hline & $11+$ & 1,36 & 54,24 & 0,21 & 29,54 & 38,39 & 46,56 & 54,24 & 61,55 & 68,56 & 75,32 \\
\hline \multirow[t]{6}{*}{ Meninas } & $6+$ & 0,37 & 27,18 & 0,29 & 14,00 & 17,79 & 22,17 & 27,18 & 32,85 & 39,21 & 46,31 \\
\hline & $7+$ & 0,43 & 31,71 & 0,29 & 16,05 & 20,60 & 25,81 & 31,71 & 38,30 & 45,62 & 53,67 \\
\hline & $8+$ & 0,63 & 38,77 & 0,29 & 18,95 & 24,98 & 31,59 & 38,77 & 46,47 & 54,68 & 63,37 \\
\hline & $9+$ & 0,95 & 46,65 & 0,25 & 23,26 & 30,97 & 38,77 & 46,65 & 54,61 & 62,62 & 70,69 \\
\hline & $10+$ & 1,19 & 51,58 & 0,22 & 27,18 & 35,66 & 43,76 & 51,58 & 59,17 & 66,58 & 73,83 \\
\hline & $11+$ & 1,36 & 54,16 & 0,20 & 29,76 & 38,49 & 46,56 & 54,16 & 61,38 & 68,32 & 75,00 \\
\hline
\end{tabular}

Figura 2. Curvas centílicas relativas à prova SL de crianças açorianas dos 6 aos 11 anos de idade.
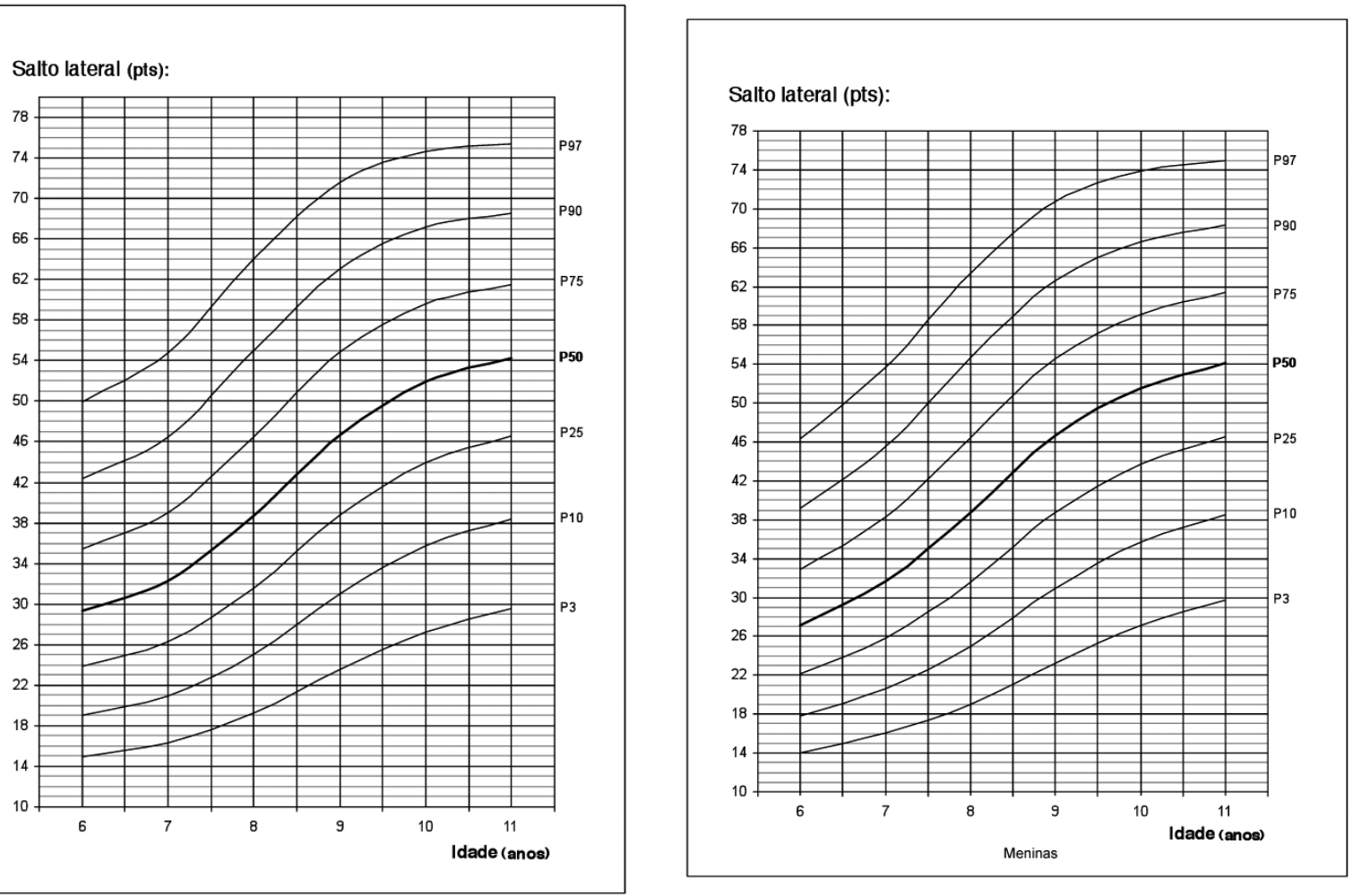
Quadro 4. Valores de LMS da prova SM de crianças açorianas de ambos os sexos.

\begin{tabular}{|c|c|c|c|c|c|c|c|c|c|c|c|}
\hline \multirow[t]{2}{*}{ Sexo } & \multirow[t]{2}{*}{ Idade } & \multirow[t]{2}{*}{$L$} & \multirow[t]{2}{*}{$M$} & \multirow[t]{2}{*}{$S$} & \multicolumn{7}{|c|}{ Percentis } \\
\hline & & & & & 3 & 10 & 25 & 50 & 75 & 90 & 97 \\
\hline \multirow[t]{6}{*}{ Meninos } & $6+$ & 0,45 & 14,43 & 0,65 & 2,07 & 4,82 & 8,91 & 14,43 & 21,44 & 30,03 & 40,24 \\
\hline & $7+$ & 0,58 & 19,81 & 0,57 & 2,99 & 7,21 & 12,9 & 19,81 & 27,96 & 37,24 & 47,60 \\
\hline & $8+$ & 0,74 & 28,36 & 0,47 & 5,57 & 12,12 & 19,80 & 28,36 & 37,66 & 47,61 & 58,13 \\
\hline & $9+$ & 0,85 & 34,79 & 0,40 & 9,03 & 17,03 & 25,67 & 34,79 & 44,30 & 54,13 & 64,24 \\
\hline & $10+$ & 0,98 & 39,88 & 0,35 & 12,39 & 21,47 & 30,64 & 39,88 & 49,17 & 58,51 & 67,88 \\
\hline & $11+$ & 1,14 & 45,32 & 0,29 & 16,92 & 26,84 & 36,25 & 45,32 & 54,13 & 62,75 & 71,20 \\
\hline \multirow[t]{6}{*}{ Meninas } & $6+$ & 0,46 & 11,78 & 0,66 & 1,54 & 3,81 & 7,21 & 11,78 & 17,57 & 24,62 & 32,97 \\
\hline & $7+$ & 0,64 & 19,05 & 0,56 & 2,61 & 6,87 & 12,42 & 19,05 & 26,62 & 35,06 & 44,30 \\
\hline & $8+$ & 0,77 & 27,56 & 0,46 & 5,40 & 11,91 & 19,37 & 27,56 & 36,34 & 45,64 & 55,38 \\
\hline & $9+$ & 0,86 & 33,71 & 0,39 & 8,75 & 16,53 & 24,90 & 33,71 & 42,86 & 52,30 & 62,00 \\
\hline & $10+$ & 0,95 & 39,12 & 0,35 & 12,38 & 21,14 & 30,07 & 39,12 & 48,28 & 57,51 & 66,82 \\
\hline & $11+$ & 1,09 & 45,62 & 0,29 & 17,78 & 27,36 & 36,60 & 45,62 & 54,47 & 63,18 & 71,77 \\
\hline
\end{tabular}

Figura 3. Curvas centílicas relativas à prova SM de crianças açorianas dos 6 aos 11 anos de idade.

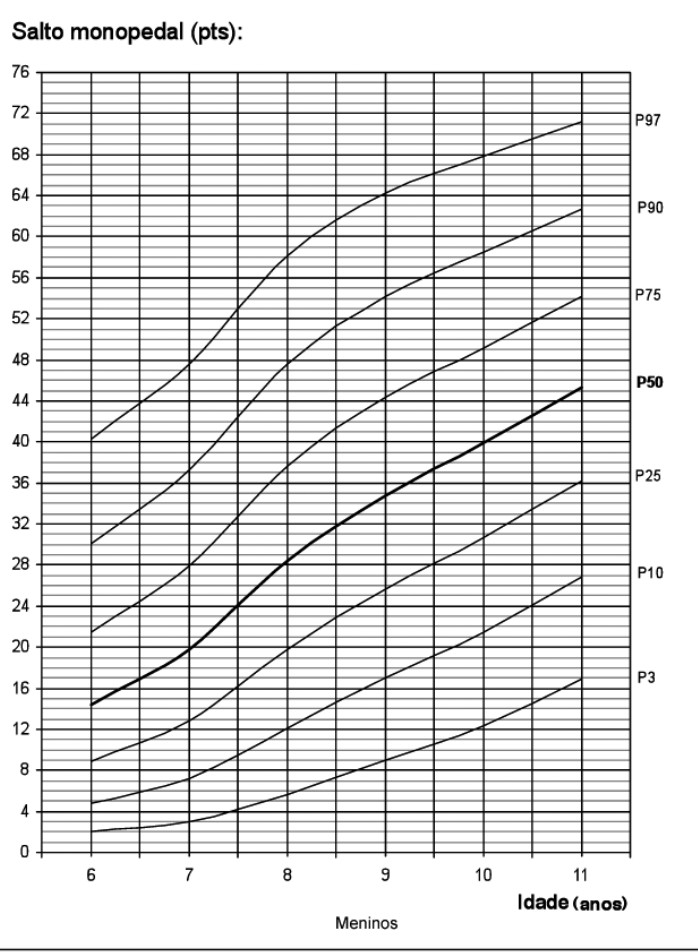

Salto monopedal (pts):

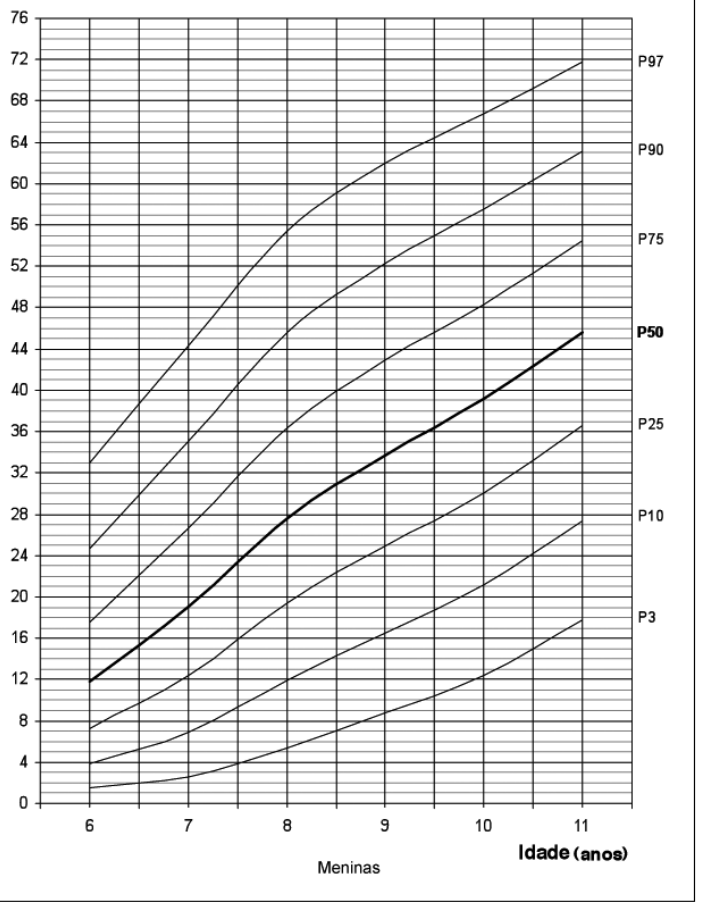


Quadro 5. Valores de LMS da prova TL de crianças açorianas de ambos os sexos.

\begin{tabular}{|c|c|c|c|c|c|c|c|c|c|c|c|}
\hline \multirow[t]{2}{*}{ Sexo } & \multirow[t]{2}{*}{ Idade } & \multirow[t]{2}{*}{$L$} & \multirow[t]{2}{*}{$M$} & \multirow[t]{2}{*}{$S$} & \multicolumn{7}{|c|}{ Percentis } \\
\hline & & & & & 3 & 10 & 25 & 50 & 75 & 90 & 97 \\
\hline \multirow[t]{6}{*}{ Meninos } & $6+$ & 1,02 & 14,01 & 0,19 & 8,41 & 10,28 & 12,15 & 14,01 & 15,86 & 17,71 & 19,56 \\
\hline & $7+$ & 0,93 & 14,95 & 0,20 & 8,98 & 10,94 & 12,93 & 14,95 & 16,98 & 19,03 & 21,09 \\
\hline & $8+$ & 0,83 & 16,72 & 0,19 & 10,39 & 12,45 & 14,56 & 16,72 & 18,94 & 21,20 & 23,51 \\
\hline & $9+$ & 0,79 & 18,08 & 0,19 & 11,64 & 13,72 & 15,87 & 18,08 & 20,34 & 22,66 & 25,03 \\
\hline & $10+$ & 0,85 & 18,89 & 0,18 & 12,36 & 14,49 & 16,67 & 18,89 & 21,15 & 23,45 & 25,79 \\
\hline & $11+$ & 0,86 & 19,05 & 0,18 & 12,51 & 14,65 & 16,83 & 19,05 & 21,30 & 23,59 & 25,91 \\
\hline \multirow[t]{6}{*}{ Meninas } & $6+$ & 1,09 & 13,39 & 0,19 & 7,98 & 9,82 & 11,62 & 13,39 & 15,15 & 16,89 & 18,61 \\
\hline & $7+$ & 0,78 & 14,65 & 0,20 & 9,02 & 10,83 & 12,71 & 14,65 & 16,66 & 18,72 & 20,83 \\
\hline & $8+$ & 0,55 & 16,53 & 0,19 & 10,65 & 12,49 & 14,45 & 16,53 & 18,74 & 21,08 & 23,53 \\
\hline & $9+$ & 0,52 & 17,83 & 0,18 & 11,89 & 13,74 & 15,72 & 17,83 & 20,07 & 22,43 & 24,91 \\
\hline & $10+$ & 0,57 & 18,79 & 0,17 & 12,79 & 14,68 & 16,68 & 18,79 & 21,01 & 23,33 & 25,75 \\
\hline & $11+$ & 0,59 & 19,15 & 0,17 & 13,15 & 15,05 & 17,05 & 19,15 & 21,35 & 23,64 & 26,02 \\
\hline
\end{tabular}

Figura 4. Curvas centílicas relativas à prova TL de crianças açorianas dos 6 aos 11 anos de idade.
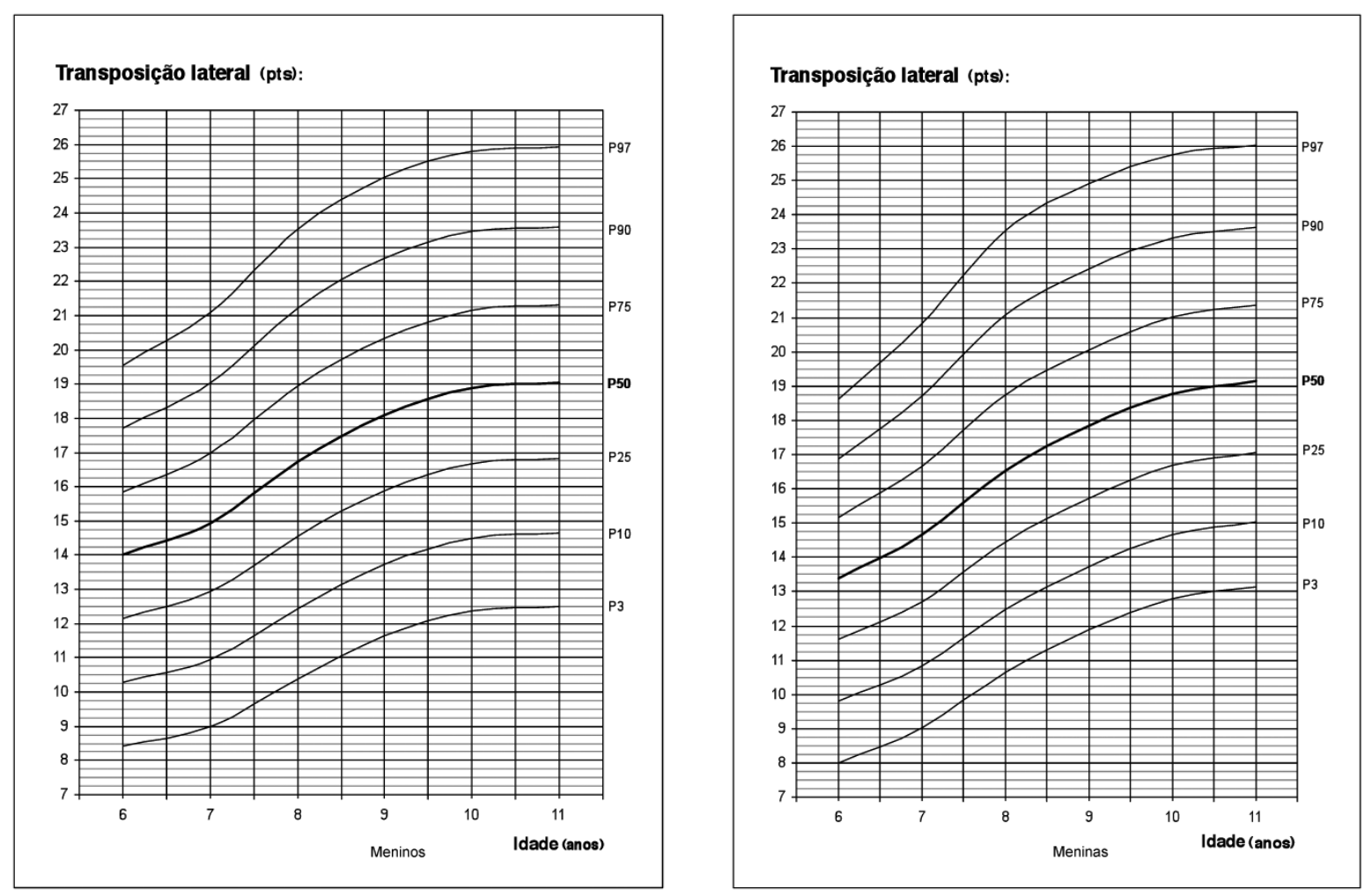
centílicas estão representadas nas Figuras 1, 2, 3 e 4. Do ponto de vista normativo, os percentis $3,10,25$, 50, 75, 90 e 97 são aceitáveis para descrever a forte variação que se encontra em cada prova. São medidas de posição relativa, que determinam, para um dado valor, a percentagem de indivíduos que se situam acima e abaixo desse valor.

Tal como seria de esperar, em todas as provas é evidente um incremento do desempenho dos valores médios e o mesmo ocorre para categorias extremas de performance, quer seja o P3 ou P10, ou ainda os P90 e P97. Este padrão é evidente nos dois sexos.

\section{DISCUSSÃO}

O grande propósito deste estudo foi construir cartas centílicas da CoM de crianças dos 6 aos 11 anos de idade da RAA a partir de uma amostra mista de informação de natureza transversal e longitudinal. Decorre daqui a necessidade de situar a discussão e relevância dos resultados numéricos e gráficos em dois planos: (1) metodológico e (2) pedagógico. Tem sido corrente, em Auxologia, a construção de cartas centílicas da altura, peso e outras medidas somáticas a partir de modelos estruturais e não estruturais $(36,28)$ com base em informação transversal $\left.^{27}, 15\right)$ e ou longitudinal $(6,13)$. Um dos modelos estatísticos mais actuais e eficientes, face à sua elegância e versatilidade, é o LMS ${ }^{4}$, , cuja aplicabilidade é corrente $(6,27,7,16)$. Tanto quanto julgamos saber, nada obsta a que seja utilizado em dados contínuos de desempenho motor que mostrem um comportamento crescente ao longo da idade. As observações relativas ao desenvolvimento coordenativo, marcado pelos quatro testes da bateria de testes KTK, das crianças açorianas prestam-se bem ao uso do modelo LMS. Do ponto de vista amostral há observações suficientes para estimar, com precisão, percentis tão extremos quanto os P3 e P97(11). Do mesmo modo, e mesmo na presença de alguma violação da normalidade da distribuição dos resultados em cada uma das provas nos valores discretos de idade das crianças dos dois sexos, a transformação Box-Cox contida no modelo permite estimar com rigor os diferentes percentis, do P3 ao P97, e construir curvas suavizadas detalhando o comportamento das suas trajectórias ao longo da idade. Os valores de L (transformação Box-Cox e respectiva curva) são a expressão da assi- metria da distribuição, sendo que valores de 1 reflectem uma distribuição Gaussiana e inferiores ou superiores a 1 assimetria à direita e à esquerda, respectivamente. Nos resultados, é notória uma tendência crescente dos valores de L para expressar a normalidade das distribuições. Este tipo de resultados é equivalente ao que ocorre noutros estudos de construção de cartas centílicas para a estatura, peso ou perímetro da cintura $(32,16,3)$. Os valores de $\mathrm{M}$ (mediana ou P50 e respectiva curva) das quatro provas apresentam trajectórias suavizadas por cubic splines salientando a sua forma crescente não linear ao longo da idade nos dois sexos.

Os resultados de $\mathrm{S}$ (coeficiente de variação e respectiva curva) são relativamente baixos para a altura (entre 0.08 e 0.30 ). Não obstante os valores obtidos nas provas do KTK serem superiores aos das medidas somáticas, são muito semelhantes aos de Bustamante(3) que também construiu cartas centílicas dos testes do KTK numa amostra da população peruana, ou aos de Rosigne et al.(32) na construção de cartas centílicas para a altura da população do país basco.

Os valores centílicos do desempenho, nas quatro provas do KTK (com excepção da prova TL) das crianças açorianas de ambos os sexos foram inferiores aos obtidos no estudo do Perú. Quando comparamos os valores obtidos, ao longo da idade, nas provas ER e SM do KTK com os resultados de Bustamante(3) verificamos que no Perú os resultados foram superiores aos do presente estudo no que respeita ao P50. Isto é, na prova ER, nos meninos peruanos o P50 situa-se entre o valor 28 e o 50 e os açorianos encontram-se entre os 22,52 e os 45,66 ; nas meninas esse valor está entre os 32 e os 50 nas peruanas e entre 25,25 e os 45,64 nas açorianas. No $\mathrm{SM}$, os valores dos rapazes peruanos encontram-se entre os 21 e os 54 e os açorianos entre os 14,43 e os 45,32. Nas meninas peruanas os valores estão entre os 17 e os 45, já as meninas açorianas estão entre o valor 11,78 e 45,62. Relativamente à prova SL os rapazes açorianos $(29,29<\mathrm{P} 50>54.24)$ foram melhores dos que os peruanos $(25<\mathrm{P} 50>50)$; o mesmo se verificou para o sexo feminino, onde as açorianas apresentaram melhores resultados $(27,18$ $<\mathrm{P} 50>54,16)$ comparativamente às peruanas $(24$ $<\mathrm{P} 50>49)$. Na prova TL foi onde encontramos dife- 
renças em cada uma das idades, quer para as meninas, quer para os meninos. À excepção do estudo de Bustamante (3) com o KTK, as únicas pesquisas a que tivemos acesso que descreve uma outra metodologia de construção de cartas centílicas, mas também baseada em cubic splines e métodos de estimação de máxima verosimilhança, foram desenvolvidas por $\operatorname{Largo}^{(21,19,20)}$ que utilizaram a bateria de testes de desenvolvimento neuromotor de Zurique, Suíça, numa amostra de 662 crianças e jovens dos 5 aos 18 anos de idade. Tanto nestes estudos como no presente, as ideias fundamentais permanecem as mesmas: (1) descrever aspectos da dinâmica do desenvolvimento motor no que se refere à coordenação motora, (2) bem como a variabilidade interindividual em cada valor discreto da idade.

Convém realçar, contudo, que os autores alemães que desenvolveram a bateria KTK tinham em mente, essencialmente, um propósito distinto do que está explícito no presente estudo e que era classificar o desempenho identificando crianças com insuficiência coordenativa para os colocar em programas especiais de recuperação. Ora todo o desiderato operativo de construção do quociente motor e sua classificação remete para uma especificidade sócio-cultural e temporal que não é a da RAA de 2007. Ao invés do pensamento dos autores alemães, e porque não dispomos actualmente de um quadro conceptual e operativo sólido baseado em processos de avaliação criterial para classificar o desempenho das crianças, pensamos ser mais adequado posicionar toda a abordagem deste estudo numa metodologia essencialmente normativa (24). Ao construir cartas centílicas e providenciar valores de desempenho coordenativo do P3 ao $\mathrm{P} 97$ de crianças que estão no $1^{\circ} \mathrm{CEB}$ estamos a dar aos professores de Educação Física (EF) um instrumento sólido e actual que muito os ajudará na compreensão do desenvolvimento coordenativo dos seus alunos.

As cartas centílicas apresentam informação de maior relevo em termos de descrição e diagnóstico de uma grande variedade de características contínuas. O que não abrangem é a possibilidade de classificar as crianças em termos coordenativos pois não há pontos de corte validados que a justifiquem. Este é o problema fulcral do uso do quociente motor. Não dispomos actualmente de nenhuma explicação subs- tantiva em termos criteriais que justifique a presença de qualquer classificação. Contudo, nada obsta a que se possa estabelecer, ainda que sem fundamentação clínica e/ou pedagógica, que os valores abaixo do percentil 10 correspondam a um desempenho coordenativo insuficiente, os valores entre o percentil 10 e 90 sejam considerados ajustados para uma dada idade e sexo, e que os valores acima do percentil 90 sejam considerados superiores. Há um outro argumento que não favorece o uso do quociente motor, ou um qualquer somatório das pontuações dos quatro testes. Dois somatórios iguais podem ser obtidos com parcelas distintas. Dado que perfis distintos podem induzir o mesmo valor compósito, é nossa posição a urgência de entender o desempenho coordenativo de cada criança como um vector e não como um escalar(24). Esta visão exige que com base nos valores centílicos do desempenho da CoM se tracem perfis configuracionais e se interprete o seu significado relativamente ao que é esperado para uma dada idade e ano de escolaridade. Ora na RAA há um conjunto vasto de informação acerca da CoM que poderá ser da maior utilidade para os professores de EF. Não obstante a argumentação anterior, pode colocar-se a seguinte questão: se o propósito fundamental da bateria de testes KTK desagua empiricamente no cálculo do quociente motor e da classificação das crianças em diferentes escalas discretas, que vão de perturbações de coordenação a coordenação muito boa, qual é o sentido da construção de cartas centílicas para cada uma das provas? A resposta situa-se a quatro níveis. Em primeiro lugar não há qualquer estudo disponível fora da Alemanha, muito menos em Portugal, que tenha pesquisado, transculturalmente, a pontuação disponibilizada pelos autores alemães, nem os seus valores de corte para classificar crianças. Em segundo lugar, e tal como referimos anteriormente, não está disponível qualquer medida critério que permita classificar o desempenho coordenativo das crianças e que sirva de base para se validarem, de modo concorrente, diferentes valores do desempenho no somatório das quatro provas. Em terceiro lugar, é exigido suporte empírico para valores de corte nas diferentes idades e sexos. Ora, esta informação essencialmente clínica não está ao nosso dispor. O recurso à teoria da detecção do sinal ou a valores distintos de utilidade 
podem ser da maior relevância nesta matéria, embora não conheçamos qualquer pesquisa neste assunto. Finalmente para referir que num programa alargado de investigação clínica, pedagógica e metodológica seja possível construir cartas centílicas para um quociente motor culturalmente específico da população portuguesa. Mas este não foi o propósito desta pesquisa.

Conforme anteriormente descrito, os resultados obtidos neste estudo encontram-se abaixo dos referidos por Bustamante ${ }^{3}$ num extracto da população peruana. São de esperar diferenças inter-populacionais cujas explicações se fundam em aspectos de natureza sócio-cultural. Tal como é bem evidente nos resultados de Graf(10) em estudos de média a longa duração há um espaço de resposta positiva de crianças para além do que é esperado do curso natural das suas histórias de vida. À EF é atribuída a grande responsabilidade de desenvolvimento psicomotor das crianças, através de múltiplas opções didáctico-metodológicas das AFD propostas, melhorando o desempenho das habilidades motoras na infância, a que se pode associar uma educação também centrada na vida saudável dos alunos. Por exemplo, nas aulas de EF, deve aperfeiçoar-se o ritmo e a aquisição de destrezas motoras, aumentando a sua complexidade que levam a uma maior variabilidade dos processos de condução motora e a uma experiência motora(14). Ora uma forma de monitorizar a eficácia de tais propostas é recorrer à atribuição de significado ao desempenho de cada criança, situando os seus resultados nas cartas centílicas e verificar se, no tempo, as mudanças são estáveis ou não. Na Escola, o professor de EF depara-se com desempenhos diversos em termos de CoM dos seus alunos, que nem sempre consegue interpretar, a não ser que tenha valores de referência. Daqui a grande utilidade das cartas centílicas. A enorme variabilidade interindividual, bem representada nas cartas de cada prova, pode ser um auxiliar precioso na diferenciação do ensino. Os resultados agora disponibilizados irão fornecer aos Professores de EF uma base adequada para interpretarem o desempenho em cada teste que descreve facetas distintas da CoM no $1^{\circ} \mathrm{CEB}$. Será, sem dúvida, um acrescento relevante para o programa oficial de AFD, não obstante salientar que o desenvolvimento da CoM deva ser conside- rada não uma matéria específica, mas sim global. Ora estamos diante de mais uma dificuldade. Não estando disponíveis informações acerca da forma de avaliação global da CoM, tão pouco da prontidão coordenativa e formas de monitorização do seu desenvolvimento, os resultados e cartas deste estudo tornam-se um auxiliar precioso à acção educativa.

\section{CONCLUSÕES}

A disponibilização de cartas centílicas e respectiva distribuição de valores da CoM segundo o sexo e idade da bateria de testes KTK, constitui uma ferramenta de reflexão, estudo e de fácil utilização por parte dos professores de EF. Espera-se o seu uso na interpretação do significado do desempenho coordenativo de crianças do $1^{\circ} \mathrm{CEB}$. Se as aulas de EF neste ciclo de ensino são plenas de sentido para cada idade, é também espectável a disponibilização de procedimentos de avaliação e controlo dos alunos para garantir um desenvolvimento que se espera adequado aos grandes propósitos do acto educativo. As cartas centílicas e respectivos valores podem ser um auxiliar precioso para o professor de EF.

\section{CORRESPONDÊNCIA}

José António Ribeiro Maia

Universidade do Porto, Faculdade de Desporto.

Laboratório de Cineantropometria e Gabinete de

Estatística Aplicada.

Rua Dr. Plácido Costa, 91

4200-450 Porto

Portugal

E-mail: jmaia@fade.up.pt 


\section{REFERÊNCIAS BIBLIOGRÁFICAS}

1. Andrade MJLA (1996). Coordenação motora. Estudo em crianças do ensino básico na Região Autónoma da Madeira. Tese de Mestrado, Universidade do Porto; FADEUP.

2. Buchner D, Miles R (2002). Seeking a Contemporary Understanding of Factors that Influence Physical Activity. American Journal of Preventive Medicine 23: 3-4.

3. Bustamante A (2007). Análisis interactivo de la coordinación motora, actividad física $y$ del índice de masa corporal en escolares peruanos. Estudio transversal en niños de ambos sexos de los 6 a los 11 años de edad. Tese de Mestrado, Universidade do Porto; FADEUP.

4. Bustamente, 2007) da idade nos dois sexos.ndo a sua forma crescente nesentam trajectmportamento das suas trajetorias ao longo dCole TJ (1990). The LMS method for constructing normalized growth standards. Eur J Clin Nutr. 44(1):45-60.

5. Cole TJ, Green PJ (1992). Smoothing reference centile curves: the LMS method and penalized likelihood. Stat Med. 11(10): 1305-19.

6. Cole TJ, Roede MJ (1999). Centiles of body mass index for Dutch children aged $0 \pm 20$ years in 1980 - a baseline to assess recent trends in obesity. Annals of Human Biology 26 (4): 303-308.

7. Eisenmann JC (2005). Waist circumference percentiles for 7 to 15 year old Austalian children. Acta Pediatric 94: 11821185.

8. Fisberg M, Pedromônico MR, Braga JAP, Ferreira AMA, Pini C, Campos SCC, Lemes SO, Silva S, Silva RS, Andrade TM (1997). Comparação do desempenho de pré-escolares, mediante teste de desenvolvimento de Denver, antes e após intervenção nutricional. Rev Ass Med 43(2): 99-104

9. Gomes MPBB (1996). Coordenação, aptidão física e variáveis do envolvimento. Estudo em crianças do $1^{\circ}$ ciclo de ensino de duas freguesias do concelho de Matosinhos. Tese de Doutoramento, Universidade do Porto; FCDEF-UP.

10. Graf C, Koch B, Falkowski G, Jouck S, Christ H, Stauenmaier K, Bjarnason-Wehrens B, Tokarski W, Dordel $S$, Predel HG (2005). Effects of a School-based intervation on BMI and Motor Abilities in childhood. Journal of Sports Science and Medicine (4): 291-299

11. Guo SS, Roche AF, Chumlea WMC, Johnson C, Kuczmarski RJ, Curtin R (2000). Statistical effects of varying sample sizes on the precision of percentile estimates. American Journal of Human Biology 12: 64-74.

12. Haywood KM, Getchell N (2005). Life Span Motor Development. $4^{\mathrm{a}}$ edição. U.S.A: Human Kinetics.

13. Hauspie RC, Wachholder A (1986). Clinical standards for growth velocity in height of Belgian boys and girls, aged 2 to 18 years. International Journal of Anthropology 1 (4): 339348.

14. Hirtz P, Schielk E (1986). O desenvolvimento das capacidades coordenativas nas crianças, nos adolescentes e nos jovens adultos. Horizonte vol. III(15): 83-88.

15. Hosseini M, Carpenter RG, Mohammad K (1999). Body Mass Index reference curves for Iran. Annals of Human Biology 26 (6): 527- 535.

16. Inokuchi M, Matsuo N, Anzo M, Takayama JI, Hasegawa T (2007). Age-dependent percentile for waist circumference for Japanese children based on the 1992-1994 cross-sectional national survey data. European Journal of Pediatrics 166 (7): $655-661$.
17. Jacinto J, Comédias J, Mira J, Carvalho L (2006). Programa de Educação Física. Ministério da Educação - Lisboa. Departamento do $3^{\circ}$ Ciclo do Ensino Básico. Vol. 6.

18. Kiphard BJ, Schilling F (1974). Körperkoordinations Test für Kinder. Beltz Test GmbH. Weinheim.

19. Largo RH, Caflisch J, Hug F, Muggli K, Molnar A, Molinari L, Sheehy A, Gasser T (2001). Neuromotor development from 5 to 18 years: Part 1: Timed performance. Developmental Medicine and Child Neurology 43: 436-443.

20. Largo RH, Caflisch JA, Hug F, Muggli K, Molnar A, Molinari L (2001). Neuromotor development from 5 to 18 years: Part 2: Associated movements. Developmental Medicine and Child Neurology 43: 444-453.

21. Largo RH, Fischer JE, Rousson V (2003). Neuromotor development from kindergarten age to adolescence: developmental course and variability. Swiss Med WKLY 133:193199.

22. Lopes VP (1997). Análise dos Efeitos de dois programas distintos de Educação Física na expressão da aptidão física, coordenação e habilidades motoras em crianças do ensino primário. Tese de Doutoramento, Universidade do Porto; FCDEF-UP.

23. Lopes VP, Maia JAR, Silva R, Seabra A, Morais F (2003). Estudo do nível de desenvolvimento da coordenação motora da população escolar (6 a 10 anos de idade) da Região Autónoma dos Açores. Revista Portuguesa Ciências do Desporto 3: 47-60.

24. Maia JAR (1996). Avaliação da Aptidão Física. Uma abordagem metodológica. Revista Horizonte Vol. XIII (73): Dossier.

25. Maia JAR, Lopes VP (2002). Estudo de crescimento somático, aptidão física, actividade física e capacidade de coordenação corporal de crianças do $1^{\circ}$ Ciclo do Ensino Básico da Região Autónoma dos Açores, DREFD- Açores, DRCT, FCDEF-UP, Eds. Porto.

26. Maia JAR, Lopes VP (2007b). Crescimento e desenvolvimento de crianças e jovens Açorianos. O que pais, professores, pediatras e nutricionistas gostariam de saber. Porto: DREFD e FADEUP.

27. McCarthy HD, Jarrett KV, Crawley HF (2001). The development of waist circumference percentiles in British children aged $5.0 \pm 16.9$ y. European Journal of Clinical Nutrition 55: 902-907.

28. Moreno LA, Fleta J, Mur L, Rodriguez G, Sarria A, Bueno M (1999). Waist circumference values in Spanish children - gender related differences. European Journal of Clinical Nutrition 53: 429-433.

29. Mota, JAPS (1991). Contributo para o desenvolvimento de programas de aulas suplementares de educação física. Estudo experimental em crianças com insuficiências de rendimento motor. Tese de Doutoramento, Universidade do Porto; FCDEF-UP.

30. Perera H (2005). Readiness for school entry: a community survey. Public Health. 119, 283-289.

31. Piek, J (2006). Infant Motor Development. USA: Human Kinetics, 2006.

32. Rosigne J, SanMartin L, Fernandez-Lopes JR, Salus S, Rebato E, Vinape A, Susanne C (2001). Smoothing centile curves of height of Basque boys and girls by the application of the LMS-method. In: Dasgrupta P, Hauspie R (eds), Perspectives in Human Growth, Development and Maturation. Dordrecht. Kluver Academic Publishers, 33-43.

33. Willimczik K (1980). Development of motor control capability (body coordination) of 6-to 10-year-old children: Results of a Longitudinal Study. In M. Ostyn; G. Beunen; J. Simons (eds.). Kinanthropometry II. Baltimore: University 
Park Press; p. 328-346.

34. Wilson PH, McKenzie BE (1998). Information processing deficits associated with developmental coordination disorder: a meta-analysis of research findings. J Child Psychol Psychiatry 39(6):829-40.

35. Zaichkowsky LD, Zaichkowsky LB, Martinek TJ (1978).

Physical Activity, motor development age and sex differences. In: Landry F, Orban WDR (eds). Motor learning, sport psychology, pedagogy and didactics of physical activity. Miami: Symposia Specialists.

36. Zonnolli R, Morgese G (1996). Waist percentiles: a simple test for atherogenic disease? Acta Pediatric 85: 1368-9. 